\title{
Assessment of Profile of Antigenicity and Immunogenicity of Eimeria maxima and Eimeria tenella through Propagative Level and Histopathological Changes in Site Infection for Exploration of Optimal Low Doses of Bivalent Chicken Coccidiosis Live Vaccine
}

\author{
Endang Suprihati ${ }^{1}$ and Muchammad Yunus ${ }^{2}$ \\ 1. Department of Veterinary Parasitology, Faculty of Veterinary Medicine, Airlangga \\ University, Surabaya \\ 2. Department of Veterinary Parasitology, Faculty of Veterinary Medicine, Airlangga \\ University, Surabaya
}

\begin{abstract}
Abstrak
Live vaccines group was the first to be studied, due to the fact that live parasites reduce further reinfection. The development of vaccine to control coccidiosis caused by E. maxima and $E$. tenella in chickens is intensifying because of the increasing threat of drug resistance to anticoccidial agents. It is important, therefore, to develop a reliable standard method for the assessment of vaccine afficacy particularly antigenicity and immunogenicity become crucial. Evaluation of profile of antigenicity and immunogenicity of E. maxima and E. tenella to some low doses can be reflected through propagative level and histopathological changes. The complete random design of research was used in this study. Sixty of two weeks old broilers were divided into four groups and each group composed 15 replications. The group 1 and group 2 were chicken group inoculated orally 5 x 102 and 1 x 103 virulent E. maxima oocysts, respectively. The group 3 and group 4 were chicken group inoculated with $2.5 \times 102$ and $5 \times 102$ virulent E. tenella oocysts, respectively. Then all chickens of group 1 and group 2 were challenged with $E$. maxima oocyst at doses of $5 \times 103$, while chicken group 3 and group 4 were challenged with E. tenella oocyst at doses of $2.5 \times 103$. Observation of research that represented antigenicity and immunogenicity was clinical sign, propagative level, histopathological changes. On primary inoculation, chicken group 1 and 3 which low doses of E. maxima and E. tenella respectively inoculated was some clinical signs such as appetite, weakness, and diarrhea were not seen on all chicken, low parasite propagative level and few histopathological changes on development site, whereas on group 2 and 4 which higher doses of E. maxima and E. tenella respectively inoculated was appeared clinical signs such as appetite, weakness, and diarrhea, moderate parasite propagative level and histopathological changes on development site. Then on challenge test, group 1 and 2 which challenged $E$. maxima and group 3 and 4 which challenged E. tenella showed there were no clinical signs such as appetite, weakness, and diarrhea on all chicken groups. For the propagative level and histopathological changes, there were no significantly differences in all chicken groups. We concluded that the low doses of virulent E. maxima and E. tenella had low propagative level and few histopathological changes effect that represents a promising strategy to prevent chickens coccidiosis particularly both species.
\end{abstract}

Keywords: antigenicity, E.tenella, E. maxima, histopthological changes, immunogenicity, propagative level 


\section{INTRODUCTION}

Live vaccines group was the first to be studied, due to the fact that live parasites reduce further reinfection. The development of vaccine to control coccidiosis caused by E. maxima and E. tenella in chickens is intensifying because of the increasing threat of drug resistance to anticoccidial agents. It is important, therefore, to develop a reliable standard method for the assessment of vaccine afficacy particularly antigenicity and immunogenicity become crucial. Evaluation of profile of antigenicity and immunogenicity of E. maxima and E. tenella to some low doses can be reflected through propagative level and histopathological changes.

\section{METHODS}

The complete random design of research was used in this study. Sixty of two weeks old broilers were divided into four groups and each group composed 15 replications. The group 1 (G1) and group 2 (G2) were chicken group inoculated orally $5 \times 10^{2}$ and $1 \times 10^{3}$ virulent E. maxima oocysts, respectively. The group 3 (G3) and group 4 (G4) were chicken group inoculated with $2.5 \times 10^{2}$ and $5 \times 10^{2}$ virulent $E$. tenella oocysts, respectively. Then 14 days post primary infection, all chickens of group 1 and group 2 were challenged with $E$. maxima oocyst at doses of $5 \times 10^{3}$, while chicken group 3 and group 4 were challenged with E. tenella oocyst at doses of $2.5 \times 10^{3}$. Observation of research that represented antigenicity and immunogenicity was clinical sign, propagative level (presented by oocyst production), histopathological changes.

\section{RESULTS}

On primary inoculation, chicken group 1 and 3 which low doses of E. maxima and $E$. tenella respectively inoculated were some clinical signs such as appetite, weakness, and diarrhea were not seen on all chicken, low parasite propagative level and few histopathological changes on development site, whereas on group 2 and 4 which higher doses of E. maxima and E. tenella respectively inoculated was appeared clinical signs such as appetite, weakness, and diarrhea, moderate parasite propagative level and histopathological changes on development site. Then on challenge test, group 1 and 2 which challenged $E$. maxima and group 3 and 4 which challenged E. tenella showed there were no clinical signs such as appetite, weakness, and diarrhea on all chicken groups. For the propagative level and histopathological changes, there were no significantly differences in all chicken groups. 


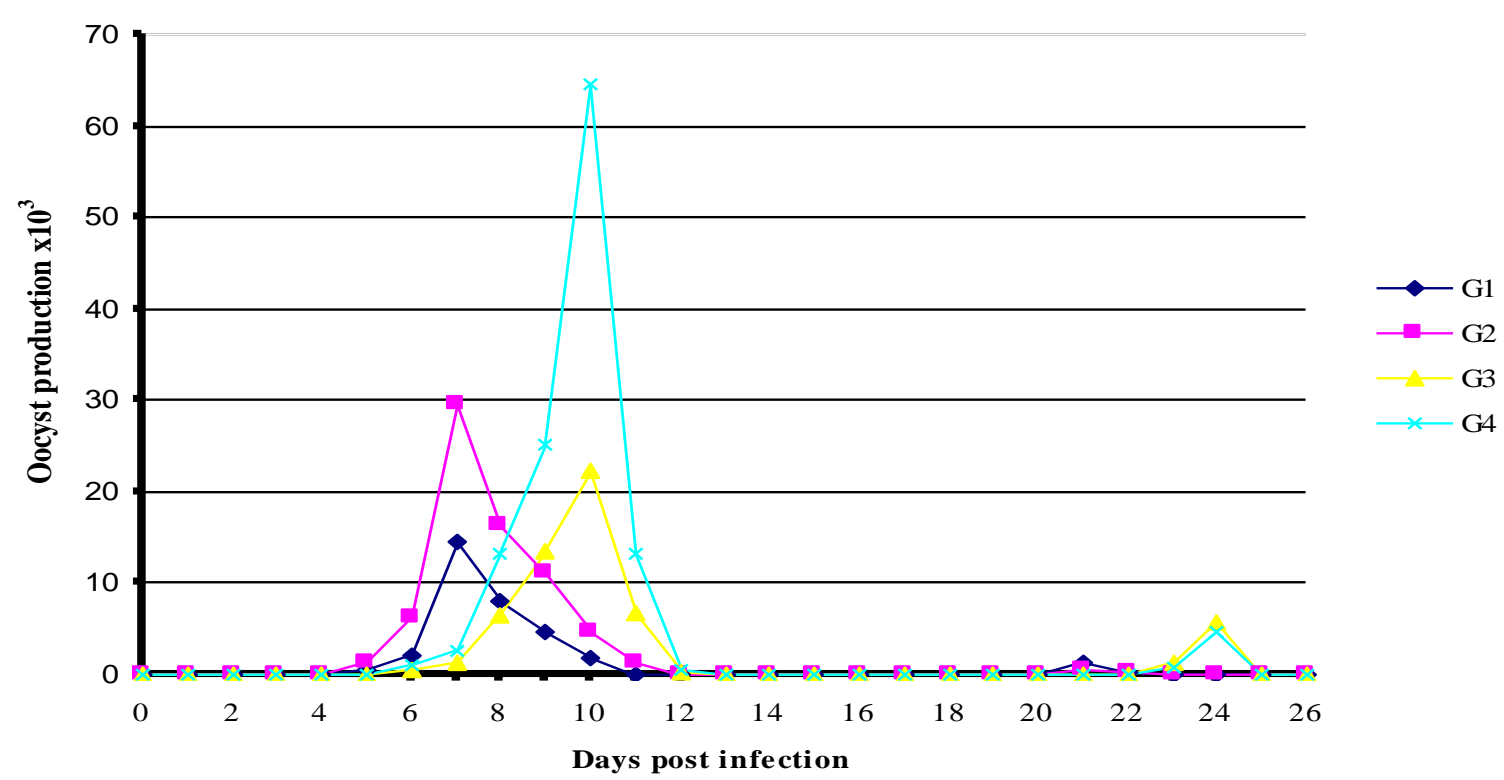

Figure 1. The pattern of daily oocyst production at each groups $(\mathrm{G} 1, \mathrm{G} 2, \mathrm{G} 3, \mathrm{G} 4)$ during primary and challenge infections, reduction of oocyst production in challenge infection at low doses of parasites (E. maxima and E. tenella) showed potencial capacity low doses in induction of protective immunity and they were not seen different with higher doses.

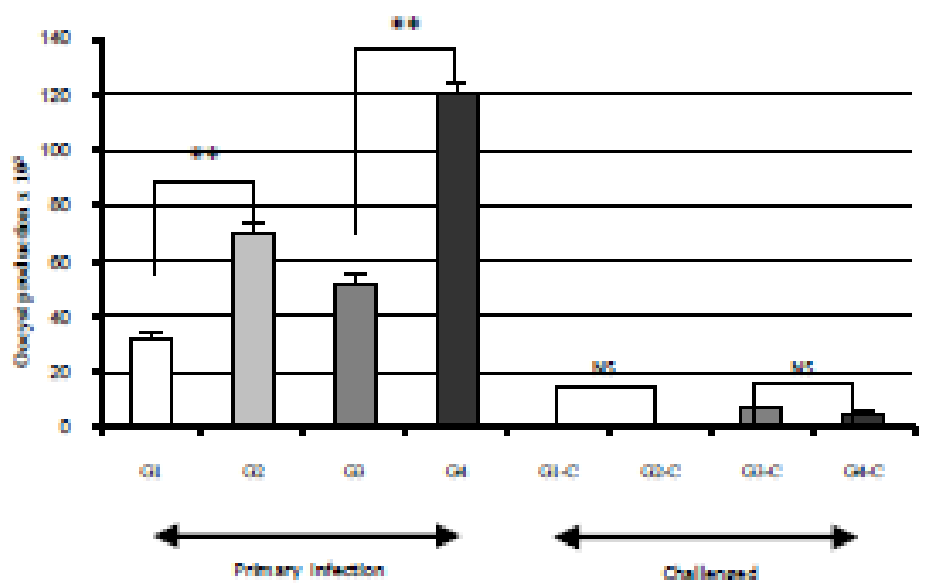

Figure 2. The comparison of totally oocyst production between low doses group (G1 and G3) and higher doses group (G2 and G4) of E. maxima and E. tenella, respectively in primary and challenge infections. There was significantly difference between low doses and higher doses at primary infection of both Eimeria sp. Reduction of oocyst production in challenge infection at low doses as well as higher doses of parasites (E. maxima and E. tenella) showed potencial capacity of low doses could express immunogenicity that can 

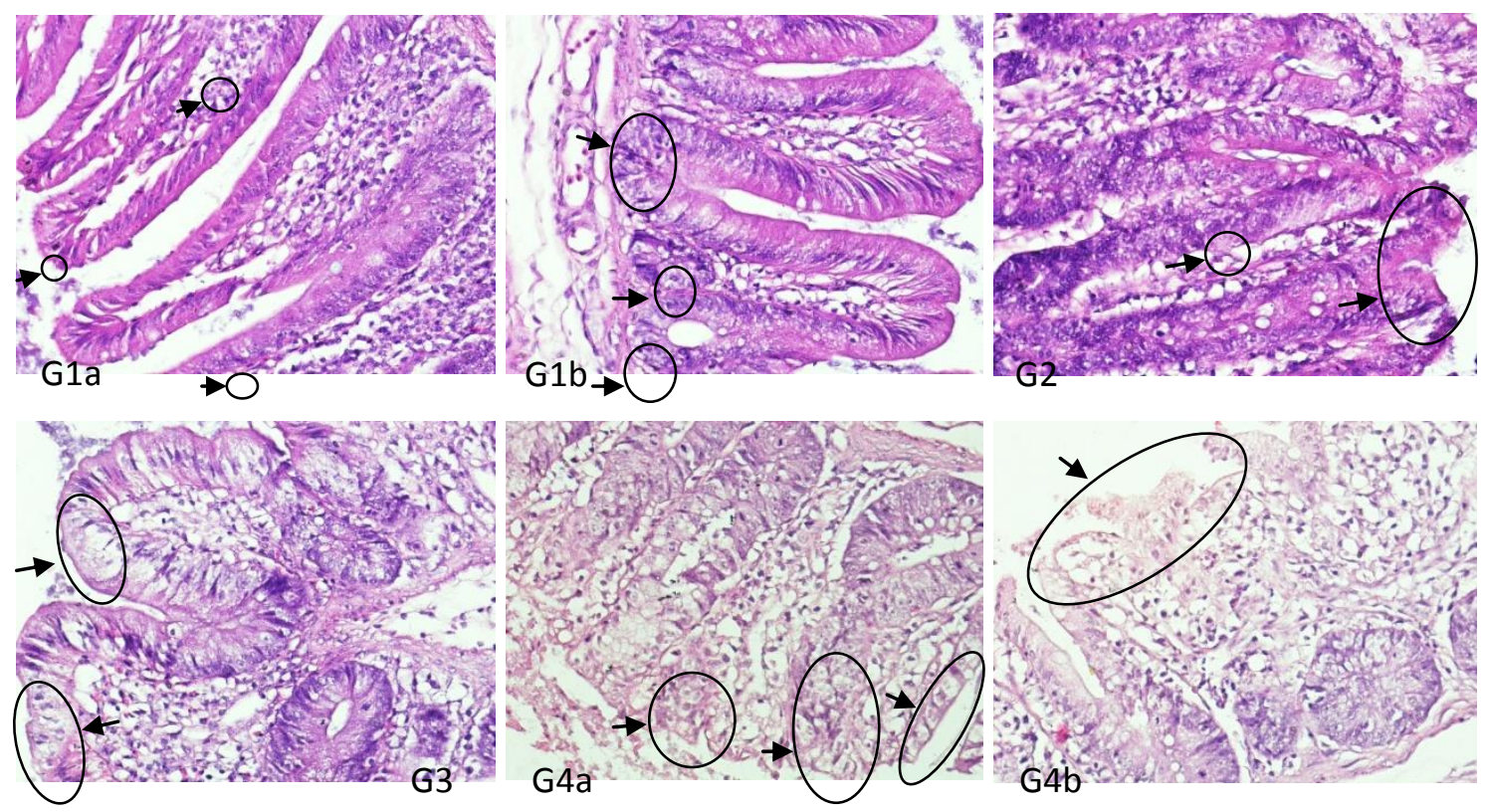

Figure 3. Histopathological changes of duodenum by E. maxima and cecum by E. tenella oocyst infected chickens. (G1a, G1b), a few endogenous development of E. maxima appeared at duodenum epithelial cells in low doses infected chickens, the same condition occurred at E. tenella in low doses infected chickens (G3), arrow. While at (G2) appeared clearly well, proliferation and multiplication of E.maxima in epithelial cell of duodenum, erosion of surface and damage of epithelial cell of duodenum due to development of E.maxima continuously, arrow. At (G4a, G4b) the crypt appeared inflammation, well development and propagation of E. tenella in site infection, erosion of mucosa surface of cecum and be accompanied many parasites proliferation.

\section{DISCUSSION}

The clinical signs of infected chicken groups at low doses of E. maxima and E. tenella were not seen during the primary infection such as dehydration, decreased appetite, diarrhea, and also dysentery, whereas at higher doses of both Eimeria sp appeared clearly those clinical signs. The low doses of each Eimeria sp were not enough to become massive propagation in site infection so that limited development and parasites were not enough to cause damage at site infection for manifestation of clinical signs. Those signs were not seen during challenge infection at low as well as higher doses, only the feces were less well formed at low dose of $E$. tenella for 1 or 2 days after challenge, but diarrhea is never evident.

Infection with one species of Eimeria induces protective immunity in the host that is long lasting and exquisitely specific to that particular parasite [1]. While a large number of inoculating oocysts is generally required to generate an immune response against Eimeria, some exceptions have been noted, e.g. E. maxima is highly immunogenic and requires only a small number of oocysts to induce almost complete immunity. In this study proved that low doses of E. maxima in primary infection can suppress propagation level by oocyst production at challenge infection. The early endogenous stages of the parasite life cycle are considered to be more immunogenic than the later sexual stages [1] although Wallach et al. [2, 3] showed 
that immunization with recombinant gamete associated antigen induced partial protection against challenge infection. Studies using oocysts irradiated to prevent intracellular development, but not invasion, demonstrated partial protection against challenge infection, thereby suggesting that sporozoites may also be immunogenic [4]

Immunity to Eimeria is stimulated by the initial developing parasite stages, particularly the schizonts, and subsequently boosted and maintained by multiple reexposure to oocysts in the litter. Thus, the recycling of infection following administration of live oocysts is critical for the development of protective immunity []․

Researchers used different criteria to evaluate coccidial infections. Some suggested that oocyst production might be a very unreliable quantitative criterion [6] as the number of oocysts produced is affected by factors such as the inherent potential of each species to reproduce in a non-immune host; immunity or resistance developed by the host; the 'crowding' factor; competition with other species of coccidian or other infectious agents; nutrition of the host; and strain differences of the host. The inherent difference in reproductive potential is high for E. tenella and E. acervulina, and low for E. maxima. Immunity, which is specific to each coccidian species, results in decreased production of oocysts after ingestion of infective oocysts [7].

In this study, higher dose of E. tenella induced damages were very severe, while $E$. maxima damages were moderate.

\section{REFERENCES}

[1] Yun, C.H., Lillehoj, H.S. and Lillehoj, E.P., 2000, Develop. Comp. Immunol., 24, 30324.

[2] Wallach, M., Pillemer, G., Yarus, S., Halabi, A,, Pugatsch, T. and Mencher, D., 1990, Infect. Immun., 58, 557- 62.

[3] Wallach, M., Smith, N.C., Petracca, M., Miller, C.M., Eckert, J. and Braun, R., 1995, Vaccine, 13, 347-54.

[4] Jenkins, M.C., Augustine, P.C., Danforth, H.D. and Barta, J.R., 1991, Infec. Immun., 59, 4042- 8 .

[5] Chapman, H. D. and Cherry, T. E., 1997, J. Appl. Poult. Res., 6 (3), 274-8

[6] Oikawa, H., Kawaguchi, H., Nakamoto, K. and Tsunoda, K., 1975, Nihon Juigaku Zasshi, 37(3), 271-9.

[7] Arabkhazaeli, F., Nabian, S., Modirsaneii, M., Mansoori, V. and Rahbari, S., 2011, Iran J. Parasitol., 6 (4), 23-32. 